\title{
Supply Function Equilibria with Capacity Constraints and Pivotal Suppliers*
}

\author{
Talat S. Genc and Stanley S. Reynolds ${ }^{\mathrm{b}}$
}

December 2008

\begin{abstract}
The concept of a supply function equilibrium (SFE) has been widely used to model generators' bidding behavior and market power issues in wholesale electricity markets. Observers of electricity markets have noted how generation capacity constraints may contribute to market power of generation firms. If a generation firm's rivals are capacity constrained then the firm may be pivotal; that is, the firm could substantially raise the market price by unilaterally withholding output. However the SFE literature has not properly analyzed the impact of capacity constraints and pivotal firms on equilibrium predictions. We characterize the set of symmetric supply function equilibria for uniform price auctions when firms are capacity constrained and show that this set is increasing as capacity per firm rises. We provide conditions under which asymmetric equilibria exist and characterize these equilibria. In addition, we compare results for uniform price auctions to those for discriminatory auctions, and we compare our SFE predictions to equilibrium predictions of models in which bidders are constrained to bid on discrete units of output.
\end{abstract}

Keywords: supply function equilibrium, pivotal firm, wholesale electricity market

JEL codes: D43, L11, L94

\footnotetext{
* We are grateful for comments and suggestions from the, participants at the Southwest Economic Theory Conference at UC Irvine, the Supply-Function Equilibrium Workshop at the University of Auckland, and seminars at Southern Methodist University, the University of Arizona and the University of Guelph.

a Department of Economics, University of Guelph, Ontario, Canada N1G 2W1, email: tgenc@uoguelph.ca

b Department of Economics, Eller College of Management, University of Arizona, Tucson, AZ 85721, email: reynolds@eller.arizona.edu
} 


\section{Introduction}

The supply function equilibrium (SFE) concept has become a widely used approach to study the exercise of market power by sellers in multi-unit auction environments. SFE models assume that each seller submits a supply function for divisible output to the auctioneer, who sets a uniform market clearing price. Klemperer and Meyer (1989) (hereafter KM) characterize supply function equilibria in environments for which product demand is uncertain. They show that there are multiple equilibria when the range of demand variation is bounded. Roughly speaking, these equilibria are contained in a range of prices between the Cournot price and the competitive price.

The SFE concept has found its widest application in the analysis of wholesale electricity auctions. Many of these auctions are run as uniform price, multi-unit auctions in which power sellers submit offer schedules indicating their willingness to supply. Examples of applications of the SFE concept to wholesale electricity markets include Green and Newbery (1992), Newbery (1998), Rudkevich, et al (1998), Baldick and Hogan (2002), and Baldick, Grant and Kahn (2004). These papers consider a variety of extensions of the KM model, including production capacity constraints, cost asymmetries, potential entry, multi-step cost functions, and forward contracting.

Recent assessments of wholesale electricity market performance have emphasized the role of the extent of excess production capacity in the market and the ability of firms to influence the market price by withholding production (see Bushnell, Knittel and Wolak (1999), Joskow and Kahn (2001), Borenstein, Bushnell and Wolak (2002), and Perekhodtsev, et al (2002)). The term "pivotal firm" has been used to describe a supplier that is able to dictate the price in the auction by withholding some portion of its production from the auction. One or more pivotal firms are most likely to be present when demand (or, load) is near its peak, when available production capacity in the market is limited relative to the peak demand, and when firms' capacities are asymmetrically distributed.

While prior applications of the SFE approach to electricity markets have considered a variety of extensions of the basic SFE model, these applications have not adequately addressed the impact of production capacity constraints nor have they examined the potential role of pivotal firms. In this paper we formulate a simple model for which the notion of a pivotal firm has a 
natural interpretation. We assume that demand is uncertain and perfectly inelastic up to a maximum price. We focus on the case in which firms' marginal costs are identical and constant up to production capacity. In the symmetric model, firms have identical capacities as well as costs. In the asymmetric model, firms have differing capacities. As in other SFE models with bounded demand variation, there is a continuum of equilibria.

The introduction of production capacity constraints into a SFE model changes the analysis in a fundamental manner. The SFE model without capacity constraints utilizes quasiconcavity of firms' profit functions and first-order necessary conditions to determine optimal price-quantity pairs along with the curvature of the equilibrium supply function. When a firm's rivals have capacity constraints the firm's profit function need not be quasi-concave in price. In addition to a locally optimal price-quantity pair associated with the SFE necessary condition, a pivotal firm may have a global profit optimum at the maximum price, or price cap. By withholding output, a pivotal firm can unilaterally move the market price to the price cap. We examine the connection between pivotal firms and the set of supply function equilibria. In symmetric and asymmetric versions of the model we show that the presence of pivotal firms alters the set of equilibria. The size of the equilibrium set depends on observable market characteristics such as the amount of industry excess capacity, the demand distribution, and the number of firms. We show that as the amount of industry excess capacity falls the set of symmetric supply function equilibria becomes smaller; the equilibria that are eliminated are the lowest-priced, most competitive equilibria. We also show that if the demand distribution is concentrated near its maximum value then there are asymmetric equilibria in which the maximum price occurs with probability one.

Section 2 reviews the relevant literature. Section 3 describes the model formulation and explains preliminary results. In section 4 we consider the role of capacity constraints and pivotal suppliers in equilibrium predictions for a symmetric model. We show how the failure of quasiconcavity of profit as a function of price changes the problem of finding an optimal supply response to rivals' supply function strategies. We provide a complete characterization of symmetric supply function equilibria for all parameter configurations with a general demand distribution, which is the main contribution of this paper. We also describe two types of asymmetric equilibria that may arise. Section 5 provides a discussion of several issues, including 
asymmetric duopoly, increasing marginal costs, a comparison of SFE results with results from discrete-units bidding models, and equilibrium selection. Section 6 concludes.

\section{Background}

\subsection{Pivotal Suppliers and Market Power}

Wholesale electricity markets are particularly vulnerable to supplier market power. These markets are often organized as auctions in which firms submit offer schedules. Buyers in these auctions typically have little or no price sensitivity, so that the price elasticity of demand is close to zero. For example, buyers may be electricity distributors that are obligated to serve whatever quantity (load) their customers request at a fixed retail price. Electricity production by power generation firms is limited by capacity constraints. In many parts of the world, wholesale markets are linked together via a transmission grid. However, the ability of buyers to acquire power from distant generation firms may be limited by transmission capacity constraints. Taken together, these characteristics of wholesale electricity markets can yield significant market power for individual firms when demand is near its peak level. By unilaterally withholding output, a firm may be able to achieve a large price increase for its output, because of the combination of zero or low demand elasticity and limited production capacity of rival firms. For example, Borenstein, Bushnell and Wolak (2002) estimate that 50 percent of electricity expenditures in California in summer 2000 could be attributed to exercise of market power by generation firms.

The concept of a pivotal firm embodies an extreme type of market power that may be present in wholesale electricity markets. Suppose that market demand is perfectly inelastic up to a maximum price, or price cap, and firms have capacity constraints. If the market demand quantity exceeds the sum of production capacities of a firm's rivals with positive probability then that firm is said to be a pivotal firm. The firm is pivotal in the sense that it can move the market price to the price cap with positive probability by withholding output at prices below the cap. We develop a model in which the pivotal firm concept plays a crucial role in determining the set of equilibria.

The Residual Supply Index (RSI) is a market power index that was devised by the California Independent System Operator (see Sheffrin (2001, 2002)). RSI is calculated as the ratio of residual supply (total supply minus largest seller's capacity) to the total demand. Using 
summer 2000 peak hourly data from the California Power Exchange, Sheffrin (2001) found a negative correlation between the Lerner Index and RSI. She finds that when RSI is about 1.2, the average price-cost markup is zero.

\subsection{Equilibrium Models}

Klemperer and Meyer (1989) formulate a model in which each seller submits a supply function for divisible output as a function of the market price. There is no private information in their model, but the level of demand is uncertain at the time sellers submit supply functions. A uniform market price is determined by the intersection of the realization of the demand function and the aggregate supply function. The necessary conditions for equilibrium yield a system of differential equations that equilibrium supply functions must satisfy. If the range of demand variation is bounded then there is a continuum of equilibria; maximum prices associated with these equilibria range from the competitive price to the Cournot price.

Green and Newbery (1992) applied the SFE model to analyze competition in the British wholesale electricity spot market. This market was run as a uniform price auction in which power sellers submit offer schedules. Green and Newbery argue that in a symmetric model, firms should select the symmetric equilibrium that yields the highest profit. Using demand and cost parameters to reflect conditions in the early years of the England and Wales wholesale electricity market, they show that at the most profitable symmetric SFE, the two dominant bulk electricity firms were predicted to choose supply functions that yield prices above marginal costs and cause substantial deadweight losses. Their SFE predicted prices were well above observed prices. Wolfram (1999) performs a more detailed analysis of pool outcomes in the England and Wales market. She also finds that the most profitable symmetric SFE yields predicted prices that are substantially above actual pool prices.

Green and Newbery (1992), Newbery (1998), and Baldick and Hogan (2002) formulate SFE models that include production capacity constraints for firms. They argue, quite correctly, that if a solution of the SFE differential equation system violates any firm's capacity constraint, then that solution cannot be a SFE (see Green and Newbery (1992, pp. 938-939) and Baldick and Hogan (2002, p. 12)). However, these papers neglect another effect of capacity constraints. Even when capacity constraints are non-binding for all firms at market clearing prices for a proposed 
vector of supply function strategies, these constraints have an impact on a firm's profitability of changing its supply function. A firm may find it profitable to change its supply by withholding output and bidding up the price, given that its rivals are capacity constrained. Prior analyses of SFE models have focused on local optimality conditions for a firm's supply response to rivals' supply strategies. Local optimality conditions yield the system of differential equations that a SFE must satisfy. However, when there are capacity constraints it is important to check for global optimality of a firm's supply response. Rivals' capacity constraints may yield a non-quasiconcave payoff function for which local optima need not be globally optimal. We develop the implications of this observation in Section 4 below.

Using a formulation similar to ours, Holmberg (2008) assumes that demand exceeds total capacity with positive probability and shows that there is a unique symmetric SFE with supply functions that reach the price cap when output is equal to capacity. Our analysis assumes that demand is stochastic but never exceeds total industry capacity.. In Section 4 we show that the set of symmetric equilibria shrinks as total capacity falls, though in general, multiple equilibrium remain. Our assumption that total capacity exceeds peak demand permits our results to be compared to results from discrete-units bidding models of electricity markets, such as Fabra, et al (2006).

Delgado and Moreno (2004) provide another avenue for eliminating multiplicity of equilibria in a SFE model. They show that the only equilibrium in a supply function model with a single, certain demand function is the equilibrium that yields the Cournot price when the Coalition-proof Nash equilibrium refinement is used. However this result changes when output is subject to capacity constraints. Delgado (2006) shows that the presence of sufficiently asymmetric capacity constraints may increase the number of Coalition-proof Nash equilibrium outcomes; the new outcomes involve prices below the Cournot price.

Fabra, von der Fehr and Harbord (2006) compare discriminatory versus uniform-price electricity auctions in which firms submit offers to supply one or more discrete units of output. Their model of a uniform-price auction shares many features of our model. We compare results from their discrete units analysis to those from our analysis of supply functions with infinitely divisible output in Section 5.3. 


\section{A Supply Function Equilibrium Model}

\subsection{Model Formulation}

We assume that the level of demand is $\theta$, a random variable that is independent of the market price. The level of demand is distributed according to $F(\theta)$ with support, $[\underline{\theta}, \bar{\theta}]$, where $0 \leq \underline{\theta}<\bar{\theta}$. Demand is perfectly inelastic for prices up to an exogenous market reserve price, $\bar{p}$. This reserve price could represent either buyers' maximum willingness to pay or, a price cap imposed by regulators. ${ }^{1}$

There are $n \geq 2$ firms. We assume a simple cost structure in which each firm has constant marginal cost of production, $c$, up to its capacity constraint, where $0 \leq c<\bar{p}$. The capacity constraint for firm $i$ is $K_{i}$. Total capacity for all firms is, $K \equiv \sum_{i=1}^{n} K_{i}$. Throughout most of this paper we assume that firms have equal capacities, with $K_{i}=k$ for $i=1, \ldots n$.

Each firm $i$ is assumed to choose a supply function, $s_{i}(p)$, from the set $\mathbf{S}$, which is defined as the set of non-decreasing, right continuous, and piecewise-differentiable functions mapping $\mathbf{R}_{+}$into $[0, k]$. Firms are assumed to select supply functions simultaneously prior to the realization of demand. The auctioneer (or, system operator) constructs an aggregate supply function and determines a market clearing price based on the realization of demand. Let $p(\theta)$ be the market clearing price when the level of demand is $\theta$. If firm $i$ submits $s_{i}(p)$, then $i$ is obligated to produce up to $s_{i}(p(\theta))$ when the level of demand is $\theta$ and will be paid $p(\theta)$ per unit for its production. ${ }^{2}$

Right continuity of supply functions is more general than the assumption of differentiable supply functions that is employed in most SFE analyses. ${ }^{3}$ Right continuity allows for two types

\footnotetext{
${ }^{1}$ See von der Fehr and Harbord (1993) for a discussion of market reserve prices for wholesale electricity markets.

2 Our uncertain demand formulation may also be interpreted either as a model of time-varying demand with longlived bids, as explained in Green and Newbery (1992). For instance, our model could be applied to approximate an electricity auction in which each firms submits a supply schedule for an entire day, and in which the day is divided into 48 one-half hour intervals, with a clearing price established for each one-half hour interval based on demand for each interval. See Baldick and Hogan (2002) and Fabra, et al (2006) for discussions of short-lived vs. long-lived bids in electricity auctions.

${ }^{3}$ Right continuity is the analog of left continuity of a bidder's demand function in a sales auction. Kremer and Nyborg (2004) allow bidders to use left continuous demand functions in their analysis of the effect of alternative allocation rules. Our interest is in the interaction of capacity constraints and supply strategies, rather than the impact of alternative allocation rules.
} 
of supply strategies that are important to consider when capacity constraints are present. First, it allows a strategy of offering a firm's some or all of a firm's capacity at the market reserve price. We show that this type of strategy may be part of an equilibrium when firms are capacity constrained. Second, it allows for a discontinuous jump in quantity supplied up to full capacity for prices above those on the equilibrium path of prices.

Let $S(p) \equiv \sum_{j} S_{j}(p)$ and $S_{-i}(p) \equiv S(p)-s_{i}(p)$. If $\theta>S(p)$ for $p \in[0, \bar{p}]$ then we assume that the price is $\bar{p}$ when demand is $\theta$ and $S(\bar{p})$ units are provided to buyers. Otherwise, a uniform market clearing price, $p^{m}(\theta)$, is established when demand is $\theta$ that satisfies

$$
p^{m}(\theta)=\min \{p \in[0, \bar{p}]: \theta \leq S(p)\} .
$$

It is possible that there is excess supply at a market clearing price. We assume a pro-rata on the margin (PRM) rule for allocating excess supply. This rule is commonly used in electricity auctions. Under PRM demand is first allocated to offers below the clearing price. Next, the remaining demand (quantity demanded at the clearing price less supply quantity offered at prices below the clearing price) is allocated to firms according to a pro-rata rule. If $p^{0}$ is the clearing price then the PRM rule yields sales for firm $i$ as follows:

$$
q_{i}=S_{i-}\left(p^{0}\right)+\left(\theta-S_{-}\left(p^{0}\right)\right) \frac{S_{i}\left(p^{0}\right)-S_{i-}\left(p^{0}\right)}{S\left(p^{0}\right)-S_{-}\left(p^{0}\right)}
$$

where $S_{i-}\left(p^{0}\right)=\lim _{p^{\uparrow} p^{0}} S_{i}(p)$ and $S_{-}\left(p^{0}\right)=\lim _{p^{\uparrow} p^{0}} S(p)$.

\subsection{Supply Function Equilibrium}

A supply function equilibrium (SFE) is a Nash equilibrium in supply function strategies. In this sub-section we provide preliminary results on equilibria with differentiable supply functions, under an assumption that firms' capacities are large enough so that production capacities have no impact on the set of equilibria.

Assumption A1. Each subset of $n-1$ firms has sufficient capacity to meet maximum demand. That is, $(n-1) k \geq \bar{\theta}$. 
Discontinuities in supply functions are important to consider when A1 is relaxed, as we explain in Section 4 below.

Consider the profit for firm $i$ in the event that demand is $\theta$, given that rival firm $j$ chooses supply function $s_{j}(p) \in \mathbf{S}$, for $j \neq i$. If the clearing price is $p$ and firm $i$ supplies the residual demand, $\theta-S_{-i}(p)$, then its profit is:

$$
\pi_{i}(p, \theta)=(p-c)\left[\theta-S_{-i}(p)\right]
$$

We seek a supply function $s_{i}(p)$ for firm $i$ that has the property that the clearing price $p$ maximizes $\pi_{i}(p, \theta)$ in (3.3) with $s_{i}(p)=\theta-S_{-i}(p)$, for each possible $\theta$. If its rivals' supply functions are differentiable then the necessary conditions for an (interior) optimal price for $\theta$ for each firm $i$ yield a system of ordinary differential equations for supply functions:

$$
\sum_{j \neq i} s_{j}{ }^{\prime}(p)=\frac{s_{i}(p)}{(p-c)}, i=1, \ldots, n
$$

This is a simple version of the differential equation system that has been intensively studied in the SFE literature. ${ }^{4}$

If all firms utilize a common supply function, $s(p)$, then the system of equations in (3.4) simplify to the following single differential equation:

$$
s^{\prime}(p)=\frac{s(p)}{(n-1)(p-c)}
$$

There is a continuum of solutions to (3.5) of the form:

$$
s_{i}(p)=\frac{\bar{\theta}}{n}\left(\frac{p-c}{p^{\prime}-c}\right)^{\frac{1}{n-1}}
$$

We depict these supply functions in Figure 1 . The parameter $p^{\prime}$ is a boundary price, representing the equilibrium price for the highest possible demand realization, for a particular equilibrium. $s(p)$ is a symmetric SFE strategy for each boundary price $p^{\prime} \in(c, \bar{p}]{ }^{5}$ Note that

\footnotetext{
4 This version is simple because there is no demand function term, due to perfectly inelastic demand, and because marginal cost is constant.

${ }^{5}$ Since we allow supply strategies that are right continuous, the Bertrand strategy of offering units at marginal cost, $c$, is also an equilibrium. Symmetric equilibrium strategies are of the form, offer zero units for sale for prices less than $c$, and offer $y$ units for sale for prices of $c$ or higher, where $y>\bar{\theta} /(n-1)$.
} 
the set of symmetric supply function equilibria is independent of the distribution of demand quantities.

The expected payoff for a firm when all $n$ firms choose $s(p)$ in (3.6) is:

$$
w^{s}\left(p^{\prime}\right)=\frac{\left(p^{\prime}-c\right)}{n \bar{\theta}^{n-1}} E\left[\theta^{n}\right]
$$

The supply function solutions in (3.6) are a special case of results in Rudkevich, et al (1998). They derive SFE solutions for a symmetric model with zero demand elasticity and general cost functions (including multi-step marginal cost functions).

Following Klemperer and Meyer (1989), the necessary conditions for maximization of $\pi_{i}(p, \theta)$ at each $\theta$ are also sufficient if each firm extends its supply function in (3.6) linearly in $p$ for prices above $p^{\prime}$ up to its capacity. However, this result depends critically on assumption A1. If A1 does not hold, then $\pi_{i}(p, \theta)$ need not be quasi-concave in $p$ and there may be second local maximum at a price above $p^{\prime}$. This point is developed further in section 4 .

It is possible that there are asymmetric equilibria, in addition to the symmetric equilibrium supply functions in (3.6). Let $x_{i}=s_{i}\left(p^{\prime}\right)$ be the supply quantity offered by firm $i$ at boundary price $p^{\prime}$ and let $x^{\max }$ be the largest of firms' supply quantities offered at $p^{\prime}$. Solutions of ODE system (3.4) are as follows:

$$
s_{i}(p)=\frac{\bar{\theta}}{n}\left(\frac{p-c}{p^{\prime}-c}\right)^{\frac{1}{n-1}}+\left(x_{i}-\frac{\bar{\theta}}{n}\right)\left(\frac{p^{\prime}-c}{p-c}\right)
$$

where $p^{\prime}$ and $\left(x_{1}, x_{2}, \ldots, x_{n}\right)$ satisfy,

$$
\begin{aligned}
& p^{\prime} \in(c, \bar{p}], \\
& \sum_{i=1}^{n} x_{i}=\bar{\theta} \\
& \underline{\theta} \geq \bar{\theta}\left(\sqrt[n]{\frac{n(n-1)\left(x^{\max }-\bar{\theta} / n\right)}{\bar{\theta}}}\right)
\end{aligned}
$$


The supply functions defined in (3.8) collapse to a symmetric supply function if firms have equal outputs at the boundary price. ${ }^{6}$ Condition (3.9C) is required in order for supply functions to be non-decreasing in price. If $\underline{\theta}>0$ then asymmetric supply function equilibria exist; if $\underline{\theta}=0$ then the only supply function equilibria are symmetric equilibria. ${ }^{7}$

\section{Capacity Constraints and Pivotal Suppliers}

In this section we relax the assumption that any group of $n-1$ firms has enough capacity to meet maximum demand. Instead we adopt,

Assumption A2. $(n-1) k<\bar{\theta}<n k$.

We say that firm $i$ is pivotal conditional on $\theta$ if $(n-1) k<\theta$. When this inequality holds, firm $i$ would be able to achieve a market price of $\bar{p}$ when demand is $\theta$ by submitting a supply function with some portion of its capacity bid in at price $\bar{p}$, and sell at least $\theta-(n-1) k$ units. We use the term "pivotal firm" to describe a firm that is pivotal with positive probability. In this section we focus on the symmetric case in which each firm has the same capacity; in this case, if one firm is pivotal then all are pivotal.

In what follows we examine how the presence of capacity constraints alters the set of equilibria. Consider a modified version of the strategy defined in (3.6).

$$
s^{*}(p)= \begin{cases}\frac{\bar{\theta}}{n}\left(\frac{p-c}{p^{\prime}-c}\right)^{1 /(n-1)} & \text { if } c \leq p<p^{\prime} \\ k & \text { if } p^{\prime} \leq p\end{cases}
$$

The modified supply function strategy in (4.1) defines supply quantities for prices both above and below the boundary price. In particular, (4.1) specifies that a firm produces output equal to its production capacity for prices at or above the boundary price. This is the most aggressive

\footnotetext{
${ }^{6}$ (3.8) is obtained by solving the system of linear differential equations (3.4) along with boundary conditions. For a derivation see Genc and Reynolds (2004) for two firms. They form an equilibrium since the profit functions are twice differentiable and strictly concave in price. The expression in (3.9C) obtained by taking the derivative of the supply function and equating it to zero.

${ }^{7}$ This corresponds to a result in Klemperer and Meyer (1989). In their model with downward sloping demand they show that asymmetric equilibria are ruled out if the lowest value of the demand shock yields zero quantity demanded at price equal to marginal cost.
} 
supply behavior possible for prices above the boundary price, and therefore yields the lowest possible residual demand for rival firms for prices above the boundary price. Also, note that if all $n$ firms choose the supply function (4.1) and if demand is $\bar{\theta}$ then the market clears at the boundary price, $p^{\prime}$, even though there is excess supply at price $p^{\prime}$. This is true because the limit of $s^{*}(p)$ as $p$ approaches $p^{\prime}$ from below is $\bar{\theta} / n$, and the PRM allocation rule gives first priority to supply offers below the clearing price.

Suppose that a firm's rivals adopt strategy $s^{*}(p)$, defined in (4.1) with boundary price, $p^{\prime}$. If the firm's best response is to choose $s^{*}(p)$ with the same boundary price, then this strategy is a symmetric equilibrium strategy. In a lemma used to prove Proposition 1, we show that if the firm's best response differs from $s^{*}(p)$, then its best response is of the following form:

(a) follow $s^{*}(p)$ for low prices up to some quantity, $q$,

(b) supply quantity $q$ for prices in the interval, $\left[s^{*-1}(q), \bar{p}\right)$ and,

(c) supply quantity $k$ at the price cap.

For $q \in[0, \bar{\theta}-(n-1) k]$ the expected payoff associated with this strategy is defined as,

$$
\begin{aligned}
w\left(q ; p^{\prime}\right)= & \int_{\underline{\theta}}^{\theta_{1}(q)}[(p(\theta)-c)(\theta / n)] d F(\theta)+\int_{\theta_{1}(q)}^{\theta_{2}(q)}(p(\theta)-c) q d F(\theta)+ \\
& \int_{\theta_{2}(q)}^{\theta_{3}(q)}\left(p^{\prime}-c\right) q d F(\theta)+\int_{\theta_{3}(q)}^{\bar{\theta}}[(\bar{p}-c)(\theta-(n-1) k)] d F(\theta)
\end{aligned}
$$

where $p^{\prime}$ is the boundary price associated with rivals' strategy, $s^{*}(\cdot)$, quantities $\theta_{1}, \theta_{2}, \theta_{3}$ are defined as,

$$
\begin{aligned}
& \theta_{1}(q)= \begin{cases}n q & \text { if } n q \geq \underline{\theta} \\
\underline{\theta} & \text { otherwise }\end{cases} \\
& \theta_{2}(q)= \begin{cases}q+\left(\frac{n-1}{n}\right) \bar{\theta} & \text { if } q+\left(\frac{n-1}{n}\right) \bar{\theta} \geq \underline{\theta} \\
\underline{\theta} & \text { otherwise }\end{cases} \\
& \theta_{3}(q)= \begin{cases}q+(n-1) k & \text { if } q+(n-1) k \geq \underline{\theta} \\
\underline{\theta} & \text { otherwise }\end{cases}
\end{aligned}
$$

and prices are given by, 


$$
\begin{aligned}
& p(\theta)-c=\left(p^{\prime}-c\right)\left(\frac{\theta}{\bar{\theta}}\right)^{n-1} \quad \text { for } \theta \in\left[\underline{\theta}, \theta_{1}\right), \\
& p(\theta)-c=\left(p^{\prime}-c\right)\left(\frac{(\theta-q) n}{(n-1) \bar{\theta}}\right)^{n-1} \quad \text { for } \theta \in\left[\theta_{1}, \theta_{2}\right) .
\end{aligned}
$$

The boundary price $p^{\prime}$ is included as a parameter argument for payoff function $w($ ) to emphasize the dependence of this payoff on the boundary price associated with the strategies used by rival firms. This payoff function also depends on other parameters, such as capacity per firm, the number of firms, and the demand distribution. The function $w\left(q ; p^{\prime}\right)$ is continuous and bounded in $q$ for $q \in[0, \bar{\theta}-(n-1) k]$. Therefore, $w\left(q ; p^{\prime}\right)$ attains a maximum value with respect to $q$, for each boundary price $p^{\prime} \in(c, \bar{p}]$ :

$$
v\left(p^{\prime}\right) \equiv \underset{q \in[0, \bar{\theta}-(n-1) k]}{\max } w\left(q ; p^{\prime}\right)
$$

Proposition 1. Under Assumption A2 the set of symmetric equilibrium supply functions is given by $s^{*}(\cdot)$ in (4.1) with boundary prices in the interval $[p, \bar{p}]$, where the lower bound of the interval is defined by,

$$
\underline{p}=\left\{p^{\prime} \in(c, \bar{p}]: v\left(p^{\prime}\right)=w^{s}\left(p^{\prime}\right)\right\} .
$$

Proof. See the Appendix.

Proposition 2. Under Assumption A2 the lower bound of the set of symmetric equilibrium boundary prices is decreasing in capacity per firm, $k$.

Proof. See the Appendix.

The lower bound $\underline{p}$ of the interval of equilibrium boundary prices is defined in (4.4). This lower bound is determined as the boundary price $p^{\prime}$ at which the function $v\left(p^{\prime}\right)$ crosses the function $w^{s}\left(p^{\prime}\right)$ from above. The function $w^{s}\left(p^{\prime}\right)$ indicates the payoff per firm when all firms choose supply function $s^{*}(\cdot)$ in (4.1) with boundary price $p^{\prime}$. This payoff function is independent of capacity per firm, $k$. However, an increase in $k$ does affect $v\left(p^{\prime}\right)$, since a higher 
capacity per firm reduces residual demand for any one firm at prices greater than or equal to $p^{\prime}$. A higher value of $k$ causes $v\left(p^{\prime}\right)$ to fall at each boundary price $p^{\prime}$, and this "shift down" yields a lower crossing point with $w^{S}\left(p^{\prime}\right)$.

The set of symmetric equilibria depends on the number of firms and on the distribution of demand quantities, as well as on capacity per firm. We do not have analytical results for the impact of changes in $n$ and $F(\theta)$. Instead we provide numerical results in Table 1 to illustrate the impact of changes in $n$ and $F(\theta)$ on the equilibrium set for an example. The table is based on demand distribution $F(\theta)=\theta^{a}$, for $\theta \in[0,1]$, where $a$ is a positive parameter. A larger value of parameter $a$ indicates that demand is more concentrated near its maximum possible of unity. Numerical results are based on the assumption that total capacity is held fixed at 125 percent of the maximum demand quantity.

\section{Table 1}

\section{Ratio of Minimum Boundary Markup to Maximum Markup for Symmetric Supply Function Equilibria*}

\begin{tabular}{ccccc} 
& & \multicolumn{3}{c}{$\boldsymbol{a}$ (distribution parameter) } \\
& & $\mathbf{1}$ & $\mathbf{2}$ & $\mathbf{3}$ \\
\cline { 3 - 5 } & $\mathbf{2}$ & 45.0 & 51.0 & 55.6 \\
& $\mathbf{3}$ & 17.1 & 20.5 & 23.2 \\
& $\mathbf{3}$ & & & \\
& & 4.0 & 4.7 & 5.3
\end{tabular}

* Table entries provide the value of, $(\underline{p}-c) /(\bar{p}-c)$, in percentage terms.

For each value of $n$, the lower bound of symmetric equilibrium boundary prices rises as $a$ rises. That is, as demand becomes more concentrated near its maximum value, relatively competitive supply strategies are eliminated as equilibrium strategies and the set of symmetric equilibria shrinks. Holding both total capacity and $a$ fixed, the lower bound of equilibrium boundary prices falls as $n$ increases. 
As in the model of Section 3, if $\underline{\theta}>0$ then there are asymmetric equilibria, in addition to the symmetric equilibria characterized in Proposition 1 . The introduction of capacity constraints alters the set of asymmetric equilibria in two possible ways. Consider first asymmetric supply functions defined in (3.8). Obviously, output at the boundary price must be less than capacity for each firm (so that $x^{\max } \leq k$ ). In addition, asymmetric supply functions defined by (3.8) are equilibria only for boundary prices that satisfy a condition analogous to the condition inside curly brackets in (4.4). That is, a boundary price $p^{\prime}$ associated with an asymmetric equilibrium must be high enough so that no firm would wish to switch to a different supply function that would yield clearing prices above $p^{\prime}$.

The analyses of von der Fehr and Harbord (1993) and Fabra, et al (2006) for procurement auctions with discrete units of output suggest a second type of asymmetric equilibrium that may arise. Suppose that the demand distribution is concentrated near the maximum demand quantity, so that the following assumption is satisfied.

Assumption A3. $(n-1) k<\underline{\theta}<\bar{\theta}<n k$.

Under Assumption A3, a single firm is assured of positive residual demand at any price up to the market reserve price, for each possible demand realization.

Proposition 3. Under Assumption A3 there exist asymmetric equilibria in which:

a) one firm offers no supply for prices below the market reserve price, and offers its entire capacity at the market reserve price,

b) each of the other $n-1$ firms offer their entire capacity at some price below the market reserve price, and

c) the uniform market clearing price is equal to the market reserve price for each demand realization.

Proposition 3 is based on the following two observations. First, the high-price firm has no incentive to deviate from its supply strategy as long as rival firms offer their capacity at or below price $\tilde{p}$ satisfying, $(\tilde{p}-c) k=(\bar{p}-c)(\underline{\theta}-(n-1) k)$. Second, the low-price firms have no 
incentive to deviate since each has sales equal to their capacity at the maximum possible price with probability one.

The asymmetric equilibria of Proposition 3 yield the highest possible industry profit; all potential surplus is extracted in the form of profit for firms. Note, however, that the distribution of profit may be quite uneven across firms. If the lowest possible demand quantity is only slightly greater than capacity for one firm, then the high-price firm will earn profit that is a small fraction of the profit earned by each other firm in equilibrium.

\section{Extensions and Discussion}

\subsection{Asymmetric Capacities}

Concerns about market power in wholesale electricity markets have often focused on the largest firms (e.g., see Lave and Perekhodtsev (2001)). Firms with the greatest generation capacity appear to be most likely to be able to force up the market price by withholding production. However there is relatively little analysis of models in which firms have asymmetric capacities in the SFE literature. ${ }^{9}$ An exception is a recent paper by Anderson and $\mathrm{Hu}$ (2008) that reports SFE results for particular configurations of asymmetric capacities and costs. They propose a new numerical method to calculate asymmetric SFE using an approximation technique for solving differential equations. They implement their method on several numerical examples.

We focus on duopoly markets in which firms differ only in capacities. We assume that firm 1 has more capacity than firm 2 . We also assume that $K_{1}+K_{2} \geq \bar{\theta}>K_{2}$.

If $K_{1}>K_{2} \geq \frac{1}{2} \bar{\theta}$ then symmetric supply function equilibria are feasible, since each firm has enough capacity to meet at least one-half of maximum demand. Symmetric equilibria are defined in Proposition 1, using $k=K_{2}$. Proposition 2 indicates that the lower bound of the set of symmetric equilibrium boundary prices is decreasing in $K_{2}$ for $K_{1}>K_{2}>\frac{1}{2} \bar{\theta}$.

\footnotetext{
${ }^{8}$ Note that the asymmetric equilibria of Proposition 3 rely on our definition of the set of supply strategies, which permits discontinuous supply functions.

9 Green and Newbery (1992) discuss equilibria for asymmetric duopoly. They write that it is, "... more difficult to solve for the pair of (differential) equations for the asymmetric equilibrium than the single equation for the symmetric equilibrium, and the rest of the paper will restrict attention to the symmetric case." Baldick and Hogan (2002) also consider asymmetries in capacities and cost functions. However they note that the differential equation approach of solving for supply functions may not be effective because the resulting supply functions often fail the non-decreasing property.
} 
There may also be asymmetric equilibria, depending on the value of $\underline{\theta}$. If $K_{2}<\frac{1}{2} \bar{\theta}$ then the only possible pure strategy equilibria are asymmetric. Equation (3.8) specifies smooth supply functions that can be asymmetric equilibrium strategies when firms have different outputs at the boundary price. In addition to the conditions in (3.9), strategies would need to satisfy the following two conditions in order to be asymmetric equilibrium strategies:

a) $x_{1} \leq K_{1}$ and $x_{2} \leq K_{2}$

b) The boundary price $p^{\prime}$ is high enough so that neither firm wishes to deviate to a strategy that yields prices above $p^{\prime}$.

If $K_{2}<\frac{1}{2} \bar{\theta}$ then condition (3.9C) requires that the minimum demand quantity satisfies, $\underline{\theta} \geq \sqrt{\bar{\theta}\left(\bar{\theta}-2 K_{2}\right)}$, since the smallest equilibrium output for firm one at the boundary price is, $x_{1}=\bar{\theta}-K_{2}{ }^{10}$

There may also be asymmetric equilibria in which the equilibrium price is equal to the market reserve price with probability one, as in Proposition 3. If $\underline{\theta}>K_{2}$ then there is an equilibrium in which firm one submits a supply function with zero output for prices below the market reserve price and output equal to capacity at the market reserve price. Firm two submits a supply curve such that firm one has no incentive to offer output at lower prices than firm two offers its capacity. If $\underline{\theta}>K_{1}$ then there are asymmetric equilibria in which either firm plays a "high-price” strategy and its rival plays a "low-price” strategy.

If $K_{2}<\frac{1}{2} \bar{\theta}$ then existence of a pure strategy equilibrium depends on the support of the demand distribution. Specifically, the following condition must be satisfied for existence of a pure strategy equilibrium:

$$
\underline{\theta} \geq \min \left\{K_{2}, \sqrt{\bar{\theta}\left(\bar{\theta}-2 K_{2}\right)}\right\} .
$$

If (5.1) does not hold then a pure strategy equilibrium does not exist. It is possible that mixed strategy equilibria exist, but we do not attempt to identify or characterize such equilibria.

\footnotetext{
${ }^{10}$ The expression $\underline{\theta} \geq \sqrt{\bar{\theta}\left(\bar{\theta}-2 K_{2}\right)}$ is obtained by plugging $n=2$ and $x^{\max }=\bar{\theta}-K_{2}$ into (3.9C).
} 


\subsection{Symmetric Firms with Step Marginal Costs}

In preceding sections we assumed that all firms have a common, constant marginal cost $c$ for production up to capacity. Our approach can be extended to the case in which firms have multi-step marginal cost functions. Rudkevich, et al (1998) derive SFE results for a multi-step marginal cost model with identical firms. Genc (2003) examines the impact of pivotal firms on the set of supply function equilibria when each firm has a two-step marginal cost schedule comprised of low-cost (base load) generation units and higher cost (peak load) units. We summarize two key findings from Genc (2003). First, with rising marginal cost steps, even the most competitive symmetric SFE yields positive profits since market clearing prices are above marginal cost for low cost units. As a consequence, the presence of pivotal firms may not have any effect on the set of supply function equilibria. This is in contrast to the constant marginal cost case, in which the presence of pivotal firms always eliminates the most competitive supply function equilibria (the lower bound on equilibrium markups is always positive if there are pivotal firms). Second, the fraction of total capacity that is comprised of low cost units has an impact on the set of supply function equilibria. By analyzing a series of numerical examples, Genc (2003) finds that the lower bound for the SFE initial price is non-increasing in the proportion of total capacity that is comprised of base load units.

\subsection{Offers to Supply Discrete Units vs. Supply Functions}

A supply function equilibrium formulation provides an approach for characterizing supply function strategies for infinitely divisible output in $n$-firm procurement settings. The present paper focuses on how capacity constraints and pivotal suppliers contribute to market power in a SFE model. An alternative approach is to model procurement as a $n$-firm game in which each firm submits offers to supply one or more discrete units of output. von der Fehr and Harbord (1993), Anwar (1999), and Fabra, et al (2006) utilize this approach.

We compare our results for symmetric capacities to those of Fabra, et al (2006), who consider a discrete units model of a uniform price auction with $n$ firms, constant marginal cost up to capacity, perfectly inelastic demand up to a price cap, and uncertain demand. Under Assumption A1, a discrete units model yields a unique price prediction regardless of the number of units per firm; namely, the Bertrand equilibrium with price equal to marginal cost. In contrast, 
a SFE model has multiple equilibria that include the Bertrand equilibrium as well as other equilibria with positive markups over marginal cost. Equilibria with positive markups in the divisible output model are sometimes referred to as “implicitly collusive” equilibria.

Under Assumption A3, pure strategy equilibria of the discrete units model are described in Proposition 3; these equilibria yield price equal to the market reserve price with probability one. These are the only pure strategy equilibria for the discrete units model; there are no symmetric equilibria in pure strategies. Under Assumption A3, our model with divisible output yields the asymmetric equilibria described in Proposition 3, symmetric equilibria described in Proposition 1, and other asymmetric equilibria with smooth supply functions. Equilibrium prices for the divisible output model are either equal to or lower than those for the discrete units model.

If Assumption A2 holds, but not A3, then there is not a pure strategy equilibrium for the discrete units model. If each firm supplies a single unit of output then there is a unique symmetric mixed strategy equilibrium with offers strictly above marginal cost. If each firm is permitted to submit offers for multiple units of output then there may be a mixed strategy equilibrium, but characterization or computation of such an equilibrium would appear to be quite challenging. If Assumption A2 holds, but not A3, then Proposition 1 describes symmetric equilibria of the divisible output model; in addition there may be asymmetric equilibria in smooth supply strategies. Expected payoff per firm in a symmetric equilibrium of the divisible output model is greater than or equal to expected payoff in the mixed strategy equilibrium for the single-unit offer model.

\subsection{Discriminatory vs. Uniform-price Auctions}

A discriminatory (pay-as-bid) auction format is an alternative to the uniform-price format. Under Assumption A1 there is a unique pure strategy SFE under discriminatory auction rules. The unique equilibrium involves each firm offering their capacity at a price equal to marginal cost (see Wang and Zender (2002) for the case of a sales auction). Supply function equilibria under uniform-price rules include the equilibrium with price equal to marginal cost, as well as other (implicitly collusively) equilibrium with prices greater than marginal cost. So, under Assumption A1, SFE prices for uniform-price auctions are greater than or equal to SFE prices for discriminatory auctions. 
Genc (forthcoming) examines supply function equilibria for discriminatory auctions under Assumption A2, with the additional assumption that demand is uniformly distributed. He shows that a pure strategy SFE does not exist. He characterizes a symmetric mixed strategy SFE in which firms mix over horizontal supply functions. This mixed strategy equilibrium is based on the security profit a firm can achieve by offering its capacity at the price cap; security profit is equal to: $w^{C} \equiv(\bar{p}-c) E[\max \{0, \theta-(n-1) k\}]$. Under uniform-price auction rules, a firm can also achieve profit $w^{C}$ by offering its capacity at the price cap. As a consequence, expected profit per firm in any supply function equilibrium under uniform-price auction rules is greater than or equal to expected profit per firm in equilibrium under discriminatory rules. A corollary to this profit result is that expected equilibrium prices under uniform-price rules are greater than or equal to expected equilibrium prices under discriminatory rules.

\subsection{Equilibrium Selection}

Under Assumption A1, the symmetric model has a continuum of Pareto-ranked symmetric equilibria. If the lower bound of the support of the demand distribution is positive, then there are also multiple asymmetric equilibria that, for a given vector of boundary outputs $\left(x_{1}, x_{2}, \ldots, x_{n}\right)$, are Pareto-ranked. Suppose that the amount of excess capacity is reduced, so that Assumption A2 holds and A3 does not hold. Then some equilibria are eliminated; supply strategies that yield low prices and low expected profits are no longer equilibrium strategies. If firms are able to coordinate on the most profitable supply function equilibrium then the fact that some less profitable supply strategies are eliminated as equilibria when excess capacity is reduced would be irrelevant for observed supply behavior. ${ }^{11}$ However, there are two reasons to question the ability of firms to coordinate on the most profitable SFE. First, as noted in Section 2.2, both Green and Newbery (1992) and Wolfram (1999) found that the most profitable symmetric SFE predicted prices were substantially above actual pool prices for the England and Wales wholesale electricity market. Second, evidence from laboratory experiments on coordination games shows that human subjects consistently fail to coordinate on the most profitable Nash equilibrium in some environments (see Van Huyck, et al (1990), and Devetag

\footnotetext{
${ }^{11}$ If total capacity is low enough so that a shortage occurs with positive probability, as Holmberg (2008) assumes, then there is a unique symmetric equilibrium and the selection problem disappears.
} 
and Ortmann (2007)). Failure becomes more likely as the number of players rises and the complexity of the environment increases.

Suppose that Assumption A3 holds. By Proposition 3 there are equilibria for which the price is equal to $\bar{p}$ with probability one. Expected profit for the firm that offers its supply at the market reserve price (the "high price" firm) in any such equilibrium is, $w^{H}=(\bar{p}-c)(E(\theta)-(n-1) k)$. If $w^{s}(\bar{p})>w^{H}$ then there is another equilibrium, the symmetric SFE with boundary price $\bar{p}$, that yields an expected price less than $\bar{p}$ and that is not a Pareto dominated equilibrium. $^{12}$

\section{Conclusions}

We examine the connection between capacity constraints and the set of supply function equilibria. We include production capacity constraints in our model to allow for the possibility that a single firm is pivotal; that is, the firm can unilaterally move the price to the market reserve price with positive probability by withholding output, since rivals are capacity-constrained. While other SFE analyses have considered capacity constraints, we argue that these analyses failed to properly account for the impact of these constraints due to a focus on local, rather than global, optimality conditions.

We characterize the set of symmetric supply function equilibria for a model with capacity constraints. We show that when the constraints satisfy a "pivotal supplier" condition, the set of symmetric equilibria is increasing in the amount of capacity per firm. We also show that the set of symmetric equilibria depends on the demand distribution and on the number of firms. This dependence of the equilibrium set on the demand distribution is in contrast to SFE models without capacity constraints, for which the equilibrium set is independent of the demand distribution.

We show that asymmetric equilibria exist for some configurations of the demand distribution and capacities. If the lower bound of the support of demand quantities is sufficiently high, then there are asymmetric equilibria for which the price is equal to the market reserve price with probability one.

\footnotetext{
${ }^{12}$ Whether or not this payoff inequality holds depends on $n, k$, and the demand distribution.
} 
There are a variety of possible extensions of our analysis. More general cost functions that better approximate costs of electricity generation from various plant types could be introduced. We could also consider the role that price-sensitive demand might play. We conjecture that the spirit of our results would carry through to more general models. That is, production capacity constraints shape the set of equilibrium supply functions via their role in limiting rivals' ability to respond to a firm's high price-low output supply strategy. Although our model provides some tools to assess market power issues in power markets, we also did not analyze the likely effects of other factors such as transmission constraints, and uncertain congestion and outages on supply function equilibrium predictions in the presence of pivotal suppliers. These issues are directions for future research. 


\section{Appendix}

The following lemma is useful for characterizing the set of symmetric supply function equilibria.

Lemma. Suppose that rivals of firm one choose supply function $s^{*}(\cdot)$ in (4.1) for some $p^{\prime} \in(c, \bar{p})$. Let $\hat{s}(\cdot)$ be a supply function chosen from set $\mathbf{S}$ by firm one, and define quantity $q^{\prime}$ by, $q^{\prime} \equiv \hat{s}\left(p^{\prime}\right)$.

a) If $\bar{\theta}-(n-1) k \leq q^{\prime} \leq k$ then expected profit associated with $\hat{s}(\cdot)$ for firm one is less than or equal to expected profit associated with $s^{*}(\cdot)$ for firm one.

b) If $0 \leq q^{\prime}<\bar{\theta}-(n-1) k$ then expected profit associated with $\hat{s}(\cdot)$ for firm one is less than or equal to expected profit associated with $\tilde{s}\left(p ; q^{\prime}\right) \in \mathbf{S}$, where:
(A1) $\tilde{s}\left(p ; q^{\prime}\right)=\left\{\begin{array}{l}s^{*}(p) \\ q^{\prime} \\ k\end{array}\right.$

$$
\begin{gathered}
\text { for } c \leq p \leq s^{*^{-1}}\left(q^{\prime}\right) \\
s^{*^{-1}}\left(q^{\prime}\right)<p<\bar{p} \\
\bar{p} \leq p
\end{gathered}
$$

Before providing a proof of the lemma, we explain its economic intuition. Part a of the Lemma deals with the case in which firm one's supply function $\hat{s}(\cdot)$ is high enough that market clearing prices are less than or equal to boundary price $p^{\prime}$ for all demand realizations. Given that firm one's rivals all choose $s^{*}(\cdot)$, the choice of $s^{*}(\cdot)$ by firm one will yield prices that maximize firm one's profit at each quantity $\theta$, subject to the constraint that prices are in the set $\left[0, p^{\prime}\right]$. As a consequence, expected profit for firm one associated with $s^{*}(\cdot)$ must be at least as high as expected profit associated with $\hat{s}(\cdot)$.

Part b of the Lemma deals with the case in which firm one's supply function $\hat{s}(\cdot)$ is low enough that market clearing prices are greater than the boundary price $p^{\prime}$ for at least some values of $\theta$. The situation is depicted in Figure 3, where $\hat{s}(\cdot)$ is drawn as an upward sloping supply curve through $\left(q^{\prime}, p^{\prime}\right)$. Residual demand for firm one has zero price elasticity for prices above $p^{\prime}$ up to $\bar{p}$. Firm one could increase its profit for demand realizations with clearing prices above $p^{\prime}$ by pushing the price to the reserve price, $\bar{p}$. Firm one can achieve this by 
adjusting its supply curve so that it is vertical at $q^{\prime}$ for prices greater than $p^{\prime}$ up to $\bar{p}$, and offering its entire capacity for prices at or above $\bar{p}$. For prices below $p^{\prime}$, the most profitable $(q, p)$ pairs lie along the supply curve $s^{*}(\cdot)$. However, some of these points involve quantities greater than $q^{\prime}$; these points would violate the constraint that the adjusted supply function, $\tilde{s}\left(p ; q^{\prime}\right)$, be non-decreasing in $p$. Firm one can increase its profit, relative to profit under $\hat{s}(\cdot)$, by extending its supply function vertically down from $\left(q^{\prime}, p^{\prime}\right)$ until the vertical segment intersects $s^{*}(\cdot)$, and by following $s^{*}(\cdot)$ for prices below this intersection point.

The example above demonstrates the potential complexity associated with finding the most profitable supply function in response to rivals' choice of a potential symmetric SFE strategy, $s^{*}(\cdot)$. Finding such a best-response supply function may be a daunting problem. ${ }^{13}$ However, the Lemma implies that the form of a best-response supply function when rivals choose $s^{*}(\cdot)$ has only two possibilities in our model. One possibility is that $s^{*}(\cdot)$ is a best response to rivals' choice of $s^{*}(\cdot)$; in this event $s^{*}(\cdot)$ is a symmetric SFE strategy. The second is that a supply function of the form $\tilde{s}\left(p ; q^{\prime}\right)$ in (4.2) is a best response to rivals' choice of $s^{*}(\cdot)$ ; this supply function has a vertical segment at some quantity $q^{\prime}<\bar{\theta}-(n-1) k$. Of course, in this event, $s^{*}(\cdot)$ is not a symmetric SFE strategy. This limit on the form of a best-response supply function greatly simplifies the characterization of the set of symmetric equilibria.

\section{Proof of Lemma.}

Part a) Under the hypothesis of part (a), market clearing prices associated with $\hat{s}($.$) are less than$ or equal to $p^{\prime}$ for each $\theta \in[\underline{\theta}, \bar{\theta}]$. Let $p^{*}(\theta)$ be the market clearing price at $\theta$ if all $n$ firms utilize $s^{*}(p)$ in (4.1):

$$
p^{*}(\theta)=c+\left(p^{\prime}-c\right)(\theta / \bar{\theta})^{n-1}
$$

\footnotetext{
13 See Anderson and Philpott (2002) for a characterization of the expected payoff-maximizing supply function under fairly general demand, rival supply, and cost conditions. Their analysis is not directly applicable because they assume continuity of (residual) demand in price.
} 
Given that its rivals use $s^{*}(p)$ in (4.1), the profit maximizing price for firm one, subject to the constraint that price is less than or equal to $p^{\prime}$ is $p^{*}(\theta)$ for $\theta \in[\underline{\theta}, \bar{\theta})$. The residual demand for firm one at $\theta$ if price is $p^{*}(\theta)$ is $\theta-(n-1) s^{*}\left(p^{*}(\theta)\right)=s^{*}\left(p^{*}(\theta)\right)$. So $\left(p^{*}(\theta), s^{*}\left(p^{*}(\theta)\right)\right)$ is the profit maximizing price-quantity pair for firm one at $\theta \in[\underline{\theta}, \bar{\theta})$, subject to price being no greater than $p^{\prime}$. Since market clearing prices associated with $\hat{s}($.$) are no greater than p^{\prime}$ for all $\theta$, $s^{*}(p)$ yields at least as much profit as $\hat{s}($.$) for each \theta \in[\underline{\theta}, \bar{\theta})$. Therefore, $s^{*}($.$) yields at least$ as much expected profit for firm one as $\hat{s}($.) (demand $\theta=\bar{\theta}$ has zero measure, so any difference in profit between using $s^{*}($.$) and \hat{s}($.$) at \theta=\bar{\theta}$ does not affect the expected profit comparison).

Part b) $s^{*}\left(\right.$.) used by rivals satisfies $s^{*}(p)=k$ for $p \geq p^{\prime}$. Therefore, residual demand for firm one is, $\theta-(n-1) k$, for $p \in\left[p^{\prime}, \bar{p}\right]$; residual demand is completely price inelastic over this price interval. Define quantity demanded $\hat{\theta}$ to satisfy $\hat{\theta}-(n-1) k=q^{\prime}$, or set $\hat{\theta}=\underline{\theta}$ if, $\hat{\theta}-(n-1) k>q^{\prime}$ . Market clearing prices associated with $\hat{s}\left(\right.$.) are greater than or equal to $p^{\prime}$ for $\theta>\hat{\theta}$, since there is excess demand at $p^{\prime}$ for $\theta>\hat{\theta}$. That is, $\hat{s}\left(p^{\prime}\right)+(n-1) s^{*}\left(p^{\prime}\right)=q^{\prime}+(n-1) k \leq \hat{\theta}<\theta$. So, for each $\theta>\hat{\theta}$, firm one can move the market clearing price to $\bar{p}$ with no reduction in quantity sold. This change will yield at least as much profit for firm one at demand realization $\theta$ as profit associated with $\hat{s}($.). Firm one can move market clearing prices to $\bar{p}$ for $\theta>\hat{\theta}$ by offering quantity $q^{\prime}$ for $p \in\left[p^{\prime}, \bar{p}\right)$ and quantity $k$ for $p \geq \bar{p}$. These supplies are what $\tilde{s}\left(p ; q^{\prime}\right)$ prescribes for $p \geq p^{\prime}$.

For demand realizations $\theta \leq \hat{\theta}$, market clearing prices associated with $\hat{s}($.$) are less than$ or equal to $p^{\prime}$. If market clearing prices associated with $\hat{s}\left(\right.$.) do not fall below $p^{\prime}$ then prices and firm one's profits for $\theta \leq \hat{\theta}$ are the same for $\hat{s}($.$) and \tilde{s}\left(p ; q^{\prime}\right)$. Consider the case in which market prices associated with $\hat{s}($.$) do fall below p^{\prime}$ for demand realizations $\theta \in\left[\underline{\theta}, \theta^{\prime}\right)$, where $\underline{\theta}<\theta^{\prime} \leq \hat{\theta}$. Profit for firm one for $p<p^{\prime}$ is,

$$
\pi(p, \theta)=(p-c)(\theta-R(p))
$$


where,

$$
R(p)=\frac{(n-1)}{n} \bar{\theta}\left(\frac{p-c}{p^{\prime}-c}\right)^{1 /(n-1)} .
$$

$\pi(p, \theta)$ is strictly concave in $p$ for $p \in\left[c, p^{\prime}\right]$. Fix $\theta \in\left[\underline{\theta}, \theta^{\prime}\right)$. Let $\hat{p}(\theta)$ be the market clearing price at $\theta$ if firm one uses supply function $\hat{s}(p)$. Let $p^{*}(\theta)$ be the price that maximizes $\pi(p, \theta)$ at $\theta$; the supply quantity associated with this price is $s^{*}\left(p^{*}(\theta)\right)=\theta-R\left(p^{*}(\theta)\right)$.

Case 1: $\widehat{s}(\hat{p}(\theta))=q^{\prime}<s^{*}\left(p^{*}(\theta)\right)$.

In this case, firm one would make more profit by lowering the price and raising its supply quantity at $\theta$ relative to $\hat{s}\left(\right.$.). But, since $\widehat{s}(\hat{p}(\theta))=q^{\prime}$ and $\widehat{s}\left(p^{\prime}\right)=q^{\prime}$, this change in price and quantity at $\theta$ would violate the constraint that the supply function is non-decreasing in $p$.

Case 2: $\widehat{s}(\hat{p}(\theta))<q^{\prime}<s^{*}\left(p^{*}(\theta)\right)$.

In this case, firm one could increase its quantity to $q^{\prime}$ at $\theta$ by adjusting its supply function to offer quantity $q^{\prime}$ at price $\tilde{p}(\theta)=R^{-1}\left(\theta-q^{\prime}\right)$. The price-quantity pair $\left(\tilde{p}(\theta), q^{\prime}\right)$ yields more profit for firm one at $\theta$ than does $(\hat{p}(\theta), \widehat{s}(\hat{p}(\theta)))$ due to strict concavity of $\pi(p, \theta)$ in $p$ for $p \in\left[c, p^{\prime}\right)$. For all demand realizations $\theta$ such that Case 2 applies, firm one can increase its profit by offering supply quantity $q^{\prime}$ at price $\tilde{p}(\theta)$.

Case 3: $\widehat{s}(\hat{p}(\theta))<s^{*}\left(p^{*}(\theta)\right) \leq q^{\prime}$.

In this case, firm one can achieve the profit maximizing price-quantity pair of $\left(p^{*}(\theta), s^{*}\left(p^{*}(\theta)\right)\right)$ , by lowering the price and increasing its quantity. For each demand realization such that Case 3 applies, firm one can increase its profit by utilizing supply function $s^{*}($.$) for price p^{*}(\theta)$.

Case 4: $\widehat{s}(\hat{p}(\theta))>s^{*}\left(p^{*}(\theta)\right)$.

In this case, firm one can increase its profit at $\theta$ by raising the price and reducing its quantity to $\left(p^{*}(\theta), s^{*}\left(p^{*}(\theta)\right)\right)$. 
The adjustments described in the four cases above entail offering supply quantity $q^{\prime}$ for $p \in\left(s^{*-1}\left(q^{\prime}\right), p^{\prime}\right)$ and supply quantity $s^{*}(p)$ for $p \in\left[c, s^{*-1}\left(q^{\prime}\right)\right]$. This is the supply function prescribed by $\tilde{s}\left(p ; q^{\prime}\right)$ for $p<p^{\prime}$.

\section{Proof of Proposition 1.}

A first step is to define the expected payoff associated with a strategy of the form $\tilde{s}(p ; q)$ in (A1), when rivals choose $s^{*}(\cdot)$. The payoff is a function of the quantity $q$ representing the level of output at the vertical segment of the supply function $\tilde{s}(p ; q)$. This payoff is given by the function $w\left(q ; p^{\prime}\right)$ as defined in (4.2). The function $w\left(q ; p^{\prime}\right)$ is continuous in $q$ for $q \in[0, \bar{\theta}-(n-1) k]$ and in $p^{\prime}$ for $p^{\prime} \in[c, \bar{p}]$. By the Theorem of the Maximum, $v\left(p^{\prime}\right)$ defined in is continuous in $p^{\prime}$ for $p^{\prime} \in[c, \bar{p}]$. Note that $v(c) \geq(\bar{p}-c) \int_{\underline{\theta}}^{\bar{\theta}} \max [0, \theta-(n-1) k] d F(\theta)>0=w^{s}(c)$.

If $p^{\prime}=\bar{p}$ then a firm's best response to $(n-1)$ rivals' choice of $s^{*}($.$) in (.) is price p^{*}(\theta)$ defined in (A1), and quantity $s^{*}\left(p^{*}(\theta)\right)$ at each $\theta$. This implies that $w^{s}(\bar{p}) \geq v(\bar{p})$. Also, profit

at $\bar{\theta}$ (as well as an interval of demand realizations below $\bar{\theta}$ ) associated with $p^{*}(\theta)$ and $s^{*}\left(p^{*}(\theta)\right)$ exceeds profit associated with $\tilde{s}(. ; q)$ at $\bar{\theta}$ for any $q \in[0, \bar{\theta}-(n-1) k]$. This follows because $(\bar{p}-c) \bar{\theta} / n>(\bar{p}-c)(\bar{\theta}-(n-1) k)$ by Assumption A2. So, $w^{s}(\bar{p})>v(\bar{p})$.

Since $w^{s}\left(p^{\prime}\right)$ and $v\left(p^{\prime}\right)$ are continuous in $p^{\prime}$ for $p^{\prime} \in[c, \bar{p}], w^{s}(c)<v(c)$, and $w^{s}(\bar{p})>v(\bar{p})$, there is at least one value of $p^{\prime}$ in the interval $(c, \bar{p})$ for which $w^{s}\left(p^{\prime}\right)=v\left(p^{\prime}\right)$.

Suppose that $p^{\prime} \in(c, \bar{p})$ is a boundary price for which $s^{*}($.$) is not a best response to (n-$ 1) rivals' choice of $s^{*}($.). Then there must exist some other supply function, $\widehat{s}($.$) that yields$ more profit for a firm than $s^{*}($.$) . Let \hat{p}(\theta)$ be the market clearing prices associated with $\widehat{s}($.$) .$ Let $\hat{y}$ be expected profit associated with $\widehat{s}($.$) . Now consider a boundary price p^{\prime \prime} \in\left(c, p^{\prime}\right)$. We propose new market clearing prices, defined by,

$$
\tilde{p}(\theta)=c+(\hat{p}(\theta)-c)\left(p^{\prime \prime}-c\right) /\left(p^{\prime}-c\right) .
$$


If rival firms use $s^{*}($.$) with boundary price p^{\prime \prime}$ then the quantity supplied by each rival firm at $\theta$ $s^{*}(\tilde{p}(\theta))$, is precisely the amount they would supply at $\theta$ with price $\hat{p}(\theta)$ and boundary price $p^{\prime}$. Define a new supply function,

$$
\tilde{s}(p) \equiv \widehat{s}\left(c+(p-c)\left(p^{\prime}-c\right) /\left(p^{\prime \prime}-c\right)\right) .
$$

This supply function, when played against $n-1$ rivals who use $s^{*}($.$) with boundary price p^{\prime \prime}$, yields market clearing prices $\hat{p}(\theta)$ and the same quantities as $\widehat{s}($.$) when played against rivals$ using $s^{*}($.$) with p^{\prime}$.

We know that $v\left(p^{\prime}\right) \geq \hat{y}>w^{s}\left(p^{\prime}\right)$. It is straightforward to show that expected profit associated with $\widehat{s}\left(\right.$.) with boundary price $p^{\prime \prime}$ is, $\left(\frac{p^{\prime \prime}-c}{p^{\prime}-c}\right) \hat{y}$. This implies,

$$
v\left(p^{\prime \prime}\right) \geq\left(\frac{p^{\prime \prime}-c}{p^{\prime}-c}\right) \hat{y}>\left(\frac{p^{\prime \prime}-c}{p^{\prime}-c}\right) w^{s}\left(p^{\prime}\right)=w^{s}\left(p^{\prime \prime}\right)
$$

We have shown that if $s^{*}\left(\right.$.) with boundary price $p^{\prime}$ is not a symmetric SFE then $s^{*}($.) with boundary price $p^{\prime \prime}<p^{\prime}$ is not a symmetric SFE. Therefore $v\left(p^{\prime}\right)>w^{s}\left(p^{\prime}\right)$ implies $v\left(p^{\prime \prime}\right)>w^{s}\left(p^{\prime \prime}\right)$ if $p^{\prime \prime}<p^{\prime}$, and $v\left(p^{\prime}\right)$ crosses $w^{s}\left(p^{\prime}\right)$ at a single value of $p^{\prime} \in(c, \bar{p})$, defined as $\underline{p}$. Strategy $s^{*}($.$) with boundary price p^{\prime} \in[\underline{p}, \bar{p}]$ is a symmetric SFE strategy; $s^{*}($.$) is not a$ symmetric SFE for $p^{\prime}<\underline{p}$.

\section{Proof of Proposition 2.}

The payoff function $w\left(q ; p^{\prime}\right)$ also depends on $k$, capacity per firm. $w\left(q ; p^{\prime}\right)$ is differentiable with respect to $k$ except for, $k=(\underline{\theta}-q) /(n-1)$. If $k>(\underline{\theta}-q) /(n-1)$ then:

$$
\begin{aligned}
\frac{\partial w\left(q ; p^{\prime}\right)}{\partial k} & =-(\bar{p}-c)(n-1)\left(1-F\left(\theta_{3}(q)\right)\right)-\left\{(\bar{p}-c)\left(\theta_{3}(q)-(n-1) k\right)-\left(p^{\prime}-c\right) q\right\} \frac{\partial \theta_{3}(q)}{\partial k} \\
& =-(\bar{p}-c)(n-1)\left(1-F\left(\theta_{3}(q)\right)\right)-\left(\bar{p}-p^{\prime}\right)(n-1) q<0
\end{aligned}
$$

If $k<(\underline{\theta}-q) /(n-1)$ then: 


$$
\begin{aligned}
\frac{\partial w\left(q ; p^{\prime}\right)}{\partial k} & =-(\bar{p}-c)(n-1)\left(1-F\left(\theta_{3}(q)\right)\right)-\left\{(\bar{p}-c)\left(\theta_{3}(q)-(n-1) k\right)-\left(p^{\prime}-c\right) q\right\} \frac{\partial \theta_{3}(q)}{\partial k} \\
& =-(\bar{p}-c)(n-1)<0
\end{aligned}
$$

If $k=(\underline{\theta}-q) /(n-1)$ then the left and right hand derivatives of $w\left(q ; p^{\prime}\right)$ with respect to $k$ are negative. Therefore, $w\left(q ; p^{\prime}\right)$ is strictly decreasing in $k$.

Consider the function $v\left(p^{\prime}\right)$ defined in (4.3). Since $w\left(q ; p^{\prime}\right)$ is strictly decreasing in $k$ and the constraint set $[0, \bar{\theta}-(n-1) k]$ has upper bound that is decreasing in $k$, the function $v\left(p^{\prime}\right)$ is strictly decreasing in $k$.

The lower bound of symmetric Nash equilibrium boundary prices, $\underline{p}$, defined in (4.4) is the boundary price at which $v\left(p^{\prime}\right)$ crosses $w^{s}\left(p^{\prime}\right)$ from above. An increase in $k$ reduces $v\left(p^{\prime}\right)$ for all $p^{\prime}$ and hence reduces $\underline{p}$. 


\section{References}

Anderson, E.J., and Hu, X., "Finding Supply Function Equilibria with Asymmetric Firms”, Operations Research, vol. 56, pp. 697-711, 2008.

Anderson, E.J., and Philpott, A.B., “Optimal Offer Construction in Electricity Markets”, Mathematics of Operations Research, vol. 27, pp. 82-100, 2002.

Anwar, A.W., The Case for a Discriminatory Pricing Rule in Competitive Electricity Pools, Mimeo, Department of Economics, Edinburgh University, 1999.

Baldick, R. and W. Hogan, "Capacity Constrained Supply Function Equilibrium Models of Electricity Markets: Stability, Non-decreasing Constraints, and Function Space Iterations”, University of California Berkeley, PWP-089 Working Paper, August 2002.

Baldick, R., R. Grant, and E. Kahn, "Theory and Application of Linear Supply Function Equilibrium in Electricity Markets”, Journal of Regulatory Economics, vol. 25, 2004.

Borenstein, S., J. Bushnell, and F. Wolak, "Measuring Market Inefficiencies in California's Restructured Wholesale Electricity Market”, The American Economic Review, vol. 92, December 2002.

Delgado, J. D. Moreno, “Coalition-proof Supply Function Equilibria in Oligopoly”, Journal of Economic Theory, 114, 231-254, 2004.

Delgado. J., "Coalition-proof Supply Function Equilibria Under Capacity Constraints”, Economic Theory, 29(1), 219-229, 2006.

Devetag, G., and A. Ortmann, "When and Why? A Critical Survey on Coordination Failure in the Laboratory”, Experimental Economics, 10:3, 331-344, 2007.

Fabra, N., N. von der Fehr and D. Harbord, “Designing Electricity Auctions”, Rand Journal of Economics, 37:1, 23-46, 2006.

von der Fehr, N.H., and D. Harbord, "Spot Market Competition in the UK Electricity Industry", Economic Journal, vol. 24, 1993.

Genc, T., "Some Economic Aspects of a Restructured Electricity Industry", PhD Thesis, University of Arizona, 2003.

Genc, T., "Discriminatory versus Uniform-price Electricity Auctions with Supply Function Equilibrium”, forthcoming in, Journal of Optimization Theory and Applications. 
Genc, T., and S. Reynolds, "Supply Function Equilibria with Pivotal Electricity Firms", University of Arizona Economics Department Working Paper No. 04-10, March 2004: http://w3.arizona.edu/ econ/working.htm

Green, R. and D. Newbery, "Competition in the British Electricity Spot Market”, Journal of Political Economy, v. 100, no. 51992.

Holmberg, P., "Unique supply function equilibrium with capacity constraints", Energy Economics, vol. 30, 2008.

Joskow, P., and E. Kahn, "A Quantitative Analysis of Pricing Behavior in California's Wholesale Electricity Market During Summer 2000”, Mimeo, M.I.T., 2000.

Klemperer, P., and M. Meyer, "Supply Function Equilibria in Oligopoly under Uncertainty", Econometrica, vol. 57, 1989.

Kremer, I., and K.G. Nyborg, "Divisible Good Auctions: The Role of Allocation Rules", RAND Journal of Economics, vol. 35, 2004.

Lave, L., and D. Perekhodtsev, "Capacity Withholding Equilibrium in Wholesale Electricity Markets”, mimeo, Carnegie Mellon University, 2001.

Newbery, D., "Competition, Contracts and Entry in the Electricity Spot Market”, RAND Journal of Economics, vol. 29, 1998.

Perekhodtsev, D., L. Lave, and S. Blumsack "The Pivotal Oligopoly Model for Electricity Markets”, Mimeo, Carnegie Mellon University, 2002.

Rudkevich, A., M. Duckworth, and R. Rosen, "Modeling Electricity Pricing in a Deregulated Generation Industry: The Potential for Oligopoly Pricing in a Poolco", Energy Journal, vol. 19, 1998.

Sheffrin, A. "Critical Actions Necessary for Effective Market Monitoring”, Mimeo, Department of Market Analysis, California ISO, 2001.

Sheffrin, A. "Predicting Market Power Using the Residual Supply Index" Mimeo, Department of Market Analysis, California ISO, 2002.

Wang, J. and J. Zender, “Auctioning divisible goods”, Economic Theory, vol. 19, 2002.

Wolfram, C.D. "Measuring Duopoly Power in the British Electricity Spot Market", The American Economic Review, vol.89, 1999.

Van Huyck, J., R. Battalio, and R. Beil, "Tacit Coordination Games, Strategic Uncertainty, and Coordination Failures”, The American Economic Review, vol. 80, 1990. 
Figure 1

Equilibrium Supply Functions

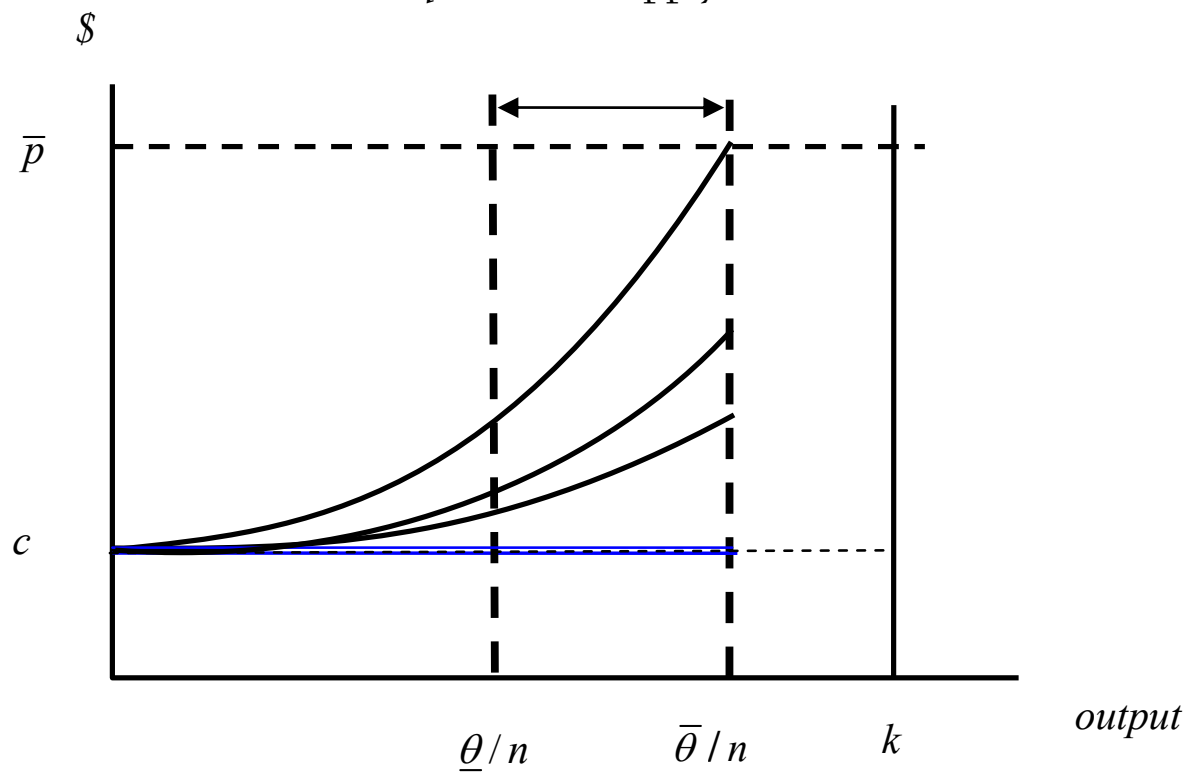

Figure 2

Illustration for Lemma, part b

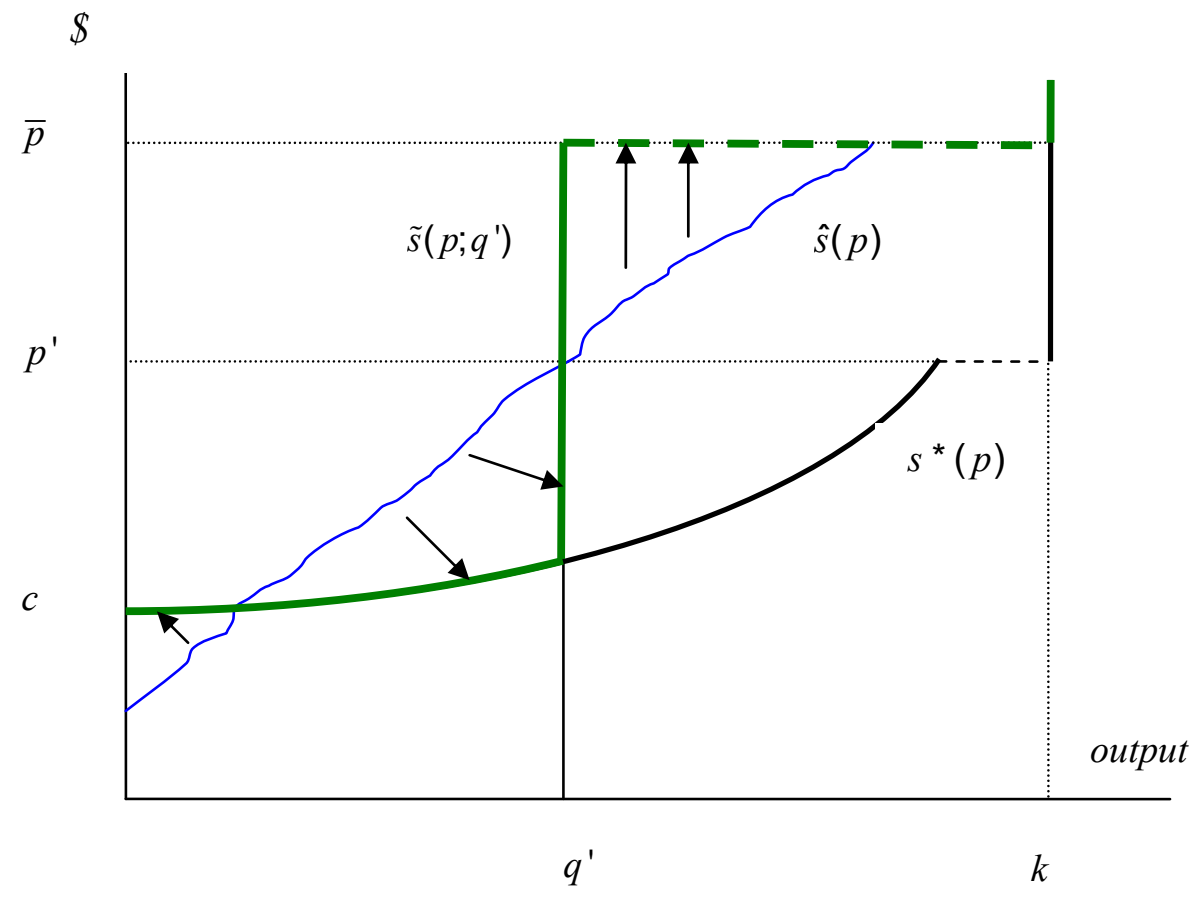

